\title{
Exploring Fringe Settings of SVMs for Classification
}

\author{
Adam Kowalczyk and Bhavani Raskutti \\ Telstra, 770 Blackburn Road, Clayton, Victoria 3168, Australia \\ \{Adam.Kowalczyk, Bhavani.Raskutti\}@team.telstra.com
}

\begin{abstract}
There are many practical applications where learning from single class examples is either, the only possible solution, or has a distinct performance advantage. The first case occurs when obtaining examples of a second class is difficult, e.g., classifying sites of "interest" based on web accesses. The second situation is exemplified by the one-class support vector machine which was the winning submission of the second task of the KDD Cup 2002.

This paper explores the limits of supervised learning using both positive and negative examples. To this end, we analyse the KDD Cup dataset using four classifiers (support vector machines and ridge regression) and several feature selection methods. Our analysis shows that there is a consistent pattern of performance differences between one and two-class learning for all algorithms investigated, and these patterns persist even with aggressive dimensionality reduction through automated feature selection. Using insight gained from the above analysis, we generate synthetic data showing similar pattern of performance.
\end{abstract}

\section{Introduction}

A standard approach for two class discrimination is to use examples from both classes to generate a model for discriminating them. This approach is so entrenched in machine learning that practitioners often will not consider data unless it contains examples of both classes. Moreover, many machine learning algorithms, such as decision trees, naive Bayes or multilayer perceptron, do not function unless the training data includes examples from two classes. However, there are many applications where obtaining examples of a second class is difficult, e.g., classifying sites of "interest" to a web surfer where the sole information that is available are the positive examples or sites that are of interest to the user. In such a case, learning from examples of one class is the only possible solution.

In addition, there are situations when the data has heavily unbalanced representatives of the two classes of interest, e.g., fraud detection and information filtering. A supervised algorithm applied to such a problem has to implement some form of balancing. In some situations, it may be beneficial to design rebalancing even more radically than warranted by unequal proportions, and ignore the large pool of negative examples and learn from positive examples only. A real life learning problem that has benefited from such an approach is the second task of the KDD Cup 2002 [4, where the winning submission learnt using just the positive examples which consisted of $<3 \%$ of the training data 8 . 
This paper explores the limits of two-class learning and analyses situations when this discrimination learning may break down. This exploration begins with an analysis of the KDD Cup dataset using four different classifiers: support vector machines and ridge regression, in several different settings. We then study the performance of these classifiers in the one-class and two-class mode when the input feature space is significantly reduced using automatic feature selection methods. The consistently better performance of the one-class models in the above analysis, leads us to a systematic study of conditions when one-class learning is advantageous. This study using synthetic data and the four classifiers used in the earlier experiments shows that data with a certain combination of properties, e.g., the presence of label noise, sparsity of features and low proportion of minority class, lends itself to better performance with one-class learners.

The paper is organised as follows. Section 2 places our research in context of existing research. Section 3 introduces the basic support vector machines in the particular form used for this research, and describes the performance measure suitable for our task. We then present results of our experiments with the KDD 2002 Cup data in Section 4.1 and that for synthetic data in Section 4.2. Finally, we discuss the implications of our results in Section 5 .

\section{Related Research}

The problem of discrimination of unbalanced classes is encountered in a large number of real life situations, e.g., detection of oil spills in satellite radar images [9], information retrieval and filtering [10] and biological domains [48]. Many solutions have been proposed to address the imbalance problem including sampling and weighting examples (cf. [7] for a thorough survey). However, they typically focus on cases when the imbalance ratio of minority to majority class is around 10:90. In this paper, we focus on extreme imbalance, where the minority class consists of around 1-3\% of the data, and extend the sampling to situations when one of the classes is ignored completely and learning is accomplished using examples from a single class.

A possibility of single class learning with support vector machines (SVM) has been noticed previously. In particular, Schölkopf et al. 14 have suggested a method of adapting the SVM methodology to one-class learning by treating the origin as the only member of the second class. This methodology has been used for image retrieval [3] and for document classification [11. In both cases, modelling is performed using examples from the positive class only, and the one-class models perform reasonably, although much worse than the two-class models learnt using examples from both classes. In contrast, in this paper, we show that for certain problems one-class models can perform better.

\section{Classifiers and Performance Metrics}

In this section we recall basic concepts of kernel machines in a form suitable for this paper. Given a training sequence $\left(x_{i}, y_{i}\right)$ of binary $n$-vectors $x_{i} \in$ $\{0,1\}^{n} \subset \mathbb{R}^{n}$ and bipolar labels $y_{i} \in\{ \pm 1\}$ for $i=1, \ldots, m$. The case of prime 
interest here is when the target class, labelled +1 , is much smaller than the background class (labelled -1 ), consisting of a minute fraction, $\approx 1-3 \%$, of the data. Our aim is to find a "good" discriminating function $f:\{0,1\}$ " $\rightarrow \mathbb{R}$ that scores the target class instances higher than the background class instances. The solution will be given in a form of a kernel machine

$$
f(x)=f^{H}(x)+b:=\sum_{i=1}^{m} \beta_{i} k\left(x, x_{i}\right)+b
$$

where $k: \mathbb{R}^{n} \times \mathbb{R}^{n} \rightarrow \mathbb{R}$ is a kernel function of one of the forms specified below and $\beta_{i}, b \in \mathbb{R}$ are parameters to be defined for the given training set as the minimiser of the regularised risk of the form as follows.

$$
\left(\beta_{i}, b\right) \mapsto\left\|f^{H}, b\right\|^{2}+\sum_{i=1}^{m} C_{y_{i}} \phi\left(1-y_{i}\left(f^{H}\left(x_{i}\right)+b\right)\right),
$$

where $C_{+1}, C_{-1} \geq 0$ are class dependent regularisation constants, $\phi: \mathbb{R} \rightarrow \mathbb{R}_{+}$is a convex loss function penalising deviations of scores from allocated labels and $\|$. $\|$ is a norm as specified below. Now we specify variations of the regularised risk (2) leading to four different cases of kernel machines used in this paper.

1. $S V M^{1}$ : For the popular support vector machine with linear penalty we use the norm $\left\|f^{H}, b\right\|^{2}:=\left\|f^{H}\right\|_{k}^{2}$, where

$$
\left\|f^{H}\right\|_{k}^{2}:=\sum_{i, j=1}^{m} \beta_{i} \beta_{j} k\left(x_{i}, x_{j}\right),
$$

and the "hinge loss" $\phi(\theta):=\max (0, \theta), \theta \in \mathbb{R}[5[15] 16]$

2. $h S V M^{1}$ : Replacing the norm in the above definition by

$$
\left\|f^{H}, b\right\|^{2}:=\left\|f^{H}\right\|_{k}^{2}+b^{2}
$$

we obtain the homogeneous support vector machine with linear penalty;

3. $h S V M^{2}$ : For the (homogeneous) support vector machine with quadratic penalty [5] we use norm (3) and the squared hinge loss $\phi(\theta):=(\max (0, \theta))^{2}$ for $\theta \in \mathbb{R}$;

4. $h R N^{2}$ : For the regularisation network [6 17] or ridge regression (c.f. [5617] we use norm (3) and the ordinary square loss $\phi(\theta):=(\theta)^{2}$ for $\theta \in \mathbb{R}$.

If the kernel $k$ satisfies the Mercer theorem assumptions [5 15]16] then for the minimiser of (2) we have $\beta_{i}=y_{i} \alpha_{i}$, where $\alpha_{i} \geq 0$ for $i=1, \ldots, m$.

In our investigations we shall be using the popular polynomial kernel

$$
k\left(x, x^{\prime}\right)=\left(x \cdot x^{\prime}\right)^{d}=\left(\sum_{i=1}^{n} \xi_{i} \xi_{i}^{\prime}\right)^{d}
$$

for $x=\left(\xi_{i}\right)$ and $x^{\prime}=\left(\xi_{i}^{\prime}\right)$ from $\{0,1\}^{n}$ and degree $d=1,2,3$ and 4 .

Note that $h S V M^{1}, h S V M^{2}$ and $h R N^{2}$ implement classifiers that correspond to separation of the data $\left(z_{i}, y_{i}\right):=\left(\Phi\left(x_{i}\right), 1, y_{i}\right) \in \mathbb{R}^{N} \times \mathbb{R} \times\{ \pm 1\}$ by a hyperplane 
in the extended feature space passing through the $(0,0) \in \mathbb{R}^{N} \times \mathbb{R}, \Phi: \mathbb{R}^{n} \rightarrow \mathbb{R}^{N}$ is a feature mapping of the observation space $\mathbb{R}^{n}$ into an appropriate Euclidean space $\mathbb{R}^{N}$ (the features space). In particular such a solution is provided also if all data points belong to a single class, i.e. if $y_{i}=$ const.

The geometrical meaning of the solution (2) can be most clearly illustrated in the limiting case of "hard margin", i.e. $C \rightarrow \infty$. In such a case, the optimal solution of (2) corresponds to the direction of the shortest vector to the convex shell spanned by all vectors $y_{i} z_{i} \in \mathbb{R}^{N} \times \mathbb{R}, i=1, \ldots, m$.

\subsection{Re-balancing of the Data}

Two way of compensation for the imbalance in the training data will be investigated in this paper.

Hard balancing. We use all $m_{+}$positive instances but randomly choose only $m_{-}$instances with the negative labels (the majority class) varying the "mixture" ratio $B_{-/+}=m_{-} / m_{+}$. We set the regularisation constants to $C_{-1}=C_{+1}=$ $C / m_{+}$, where $C>0$ is a chosen constant. In this form of balancing, $B_{-/+}=0$ is the case of positive 1-class learning, and $B_{-/+}=1$ represents the case of balanced 2-class learning when the same number of examples from both classes are used.

Soft balancing. We use all available training data but with different class regularisation constants: $C_{-1}=(1-B) C / 2 m_{-}$and $C_{-1}=(1+B) C / 2 m_{+}$, where $C>0$ and $-1 \leq B \leq+1$ is a balance parameter. Here $B=+1$ and $B=-1$ correspond to 1 -class learning, and $B=0$ is 2 -class learning with both classes "balanced" according to their prior proportions.

The advantage of the hard balancing over the soft balancing is the speed of generation of a solution, as it typically uses a smaller training set. For this reason the hard balancing was used in the most of our experiments.

\subsection{Centroids}

Now we introduce the fifth and the simplest of the five algorithms considered here in terms of generation of the solution. In contrast to SVMs, it is inherently non-sparse and so can be complex to implement in the case of non-linear kernels.

Algorithm 5, $\boldsymbol{C n t r}_{\boldsymbol{B}}$ : For the centroid classifier we set

$$
f(x)=f_{C n t r}^{H}(x):=\frac{\left((1+B) \sum_{i, y_{i}=+1} k\left(x_{i}, x\right)\right.}{2 \max \left(1, m_{+}\right)}-\frac{(1-B) \sum_{i, y_{i}=-1} k\left(x_{i}, x\right)}{2 \max \left(1, m_{-}\right)},
$$

where $x \in \mathbb{R}^{n}$ and $-1 \leq B \leq+1$ is the balance factor.

In terms of the feature space, the centroid classifier implements the projection on the direction of a weighted differences between centroids of data from both class labels. For $B=-1$ it is the direction of the majority class, for $B=+1$ that of the minority class and for $B=0$ that of the difference between centroids of both classes. 
Now we formally link centroids and SVMs. Later we shall connect this result to some of our empirical findings. The formal proof, omitted here, can be easily derived from Karush-Khun-Tucker conditions for SVM solution.

Theorem 1 If functions $k\left(x_{i},.\right), i=1, \ldots, m$ are linearly independent, then

$$
\lim _{C \rightarrow 0^{+}} \frac{f_{h S V M^{p}}^{H}}{C}=\lim _{C \rightarrow 0^{+}} \frac{f_{h R N^{2}}^{H}}{C}=f_{C n t r}^{H},
$$

for $p=1,2$, where $f^{H}$ denotes the homogeneous part of the soft balanced SVM solution (1) for the appropriate machine. Moreover, if both classes are represented in training, i.e. for the soft balance factor $-1<B<+1$, then:

$$
\lim _{C \rightarrow 0^{+}} \frac{f_{S V M^{1}}^{H}}{C}=f_{C n t r}^{H} .
$$

\subsection{Performance Measures}

We have used $A R O C$, the Area under the Receiver Operating Characteristic (ROC) curve as our main performance measure. In that we follow the steps of KDD 2002 Cup, but also, we see it as the natural metric of general goodness of classifier (as corroborated below) capable of meaningful results even if the target class is a tiny fraction of the data.

We recall that the ROC curve is a plot of the true positive rate or precision, $P\left(f\left(x_{i}\right)>\theta \mid y_{i}=1\right)$, against the false positive rate, $P\left(f\left(x_{i}\right)>\theta \mid y_{i}=-1\right)$, as a decision threshold $\theta$ is varied. The concept of ROC curve originates in the military signal detection but these days it is widely used in many other areas, including data mining, psychophysics and medical diagnosis (cf. review [2]). In the latter case, $A R O C$ is viewed as a measure of general "goodness" of a test, formalised as a predictive model $f$ in our context, with a clear statistical meaning as follows. According to Bamber's interpretation [1, $A R O C(f)$ is equal to the probability of correctly answering the two-alternative-forced-choice problem: given two cases, one $x_{i}$ from the negative and the other $x_{j}$ from the positive class, allocate scores in the right order, i.e. $f\left(x_{i}\right)<f\left(x_{j}\right)$. Additional attraction of $A R O C$ as a figure of merit is its direct link to the well researched area of order statistics via $U$-statistics and Wilcoxon-Whitney-Mann test [1].

There are some ambiguities in the case of $A R O C$ estimated from a discrete set in the case of ties, i.e. when multiple instances from different classes receive the same score. Following [1] we implement in this paper the definition

$$
\begin{array}{r}
A R O C(f)=P\left(f\left(x_{i}\right)<f\left(x_{j}\right) \mid-y_{i}=y_{j}=1\right) \\
+0.5 P\left(f\left(x_{i}\right)=f\left(x_{j}\right) \mid-y_{i}=y_{j}=1\right)
\end{array}
$$

expressing $A R O C$ in terms of conditional probabilities, which can be re-formulated in terms the rank-ordered test sequence (where the rank is imposed by the scores allocated by $f$ ).

Note that the trivial uniform random predictor has $A R O C$ of 0.5 . 


\section{Experiments}

In order to understand the boundaries when the performance of two-class classifiers deteriorate, we have explored the following datasets: (1) Real life data in the form of the Aryl Hydrocarbon Receptor signalling pathway data provided for the second task of the $2002 \mathrm{KDD}$ cup (henceforth referred to as the AHR data) (Section 4.1), and (2) Synthetic data created with some specific properties such as presence of noise in labels (Section 4.2).

\subsection{Analysis of KDD Cup 2002 Data}

In our main experiments we have used AHR-data set which is the combined training and test data sets used for task 2 of KDD Cup 2002. The data set is based on experiments by Guang Yao and Chris Bradfield of McArdle Laboratory for Cancer Research, University of Wisconsin. These experiments aimed at identification of yeast genes that, when knocked out, cause a significant change in the level of activity of the Aryl Hydrocarbon Receptor signalling pathway (cf. [4] for more details). In this paper we follow the setting of the "broad task" of the KDD Cup: the discrimination between 127 'positive' genes from the combined class encompassing the labels "change" and "control" and the remaining 4380 genes forming the 'negative' class. We note that the results for the first subtask, namely, learning "change" class are similar [8]. In our experiments this set has been repeatedly split into $70 \%$ for training and 30\% for testing. All averages and standard deviations reported are for independent tests on 20 such random splits.

Each of the 4507 instances in the data set is described by a variety of information that characterise the gene associated with the instance, e.g., associated abstracts from scientific articles,genes whose encoded proteins physically interact with one another, information about the subcellular localisation and functional classes of the proteins encoded by various genes. For the experiments described in this paper, we convert all of the information from the different files to a sparse matrix containing 18330 binary features as described in [8].

Impact of Regularisation Constant. Figure 1 shows mean AROC as a function of $C$ for four different linear kernel machines $(d=1)$ with the hard balancing (Figures A-D) and the soft balancing (Figures E-H). We use four different modes as follows: $(i)$ positive 1-class $\left(B_{-/+}=0\right.$ and $B=+1$, solid line); ( $\left.i i\right)$ negative 1-class ( $B=-1$, dotted line); (iii) balanced 2-class $\left(B_{-1+}=1\right.$ and $B=0$, dashed line); $(i v)$ un-balanced 2-class $\left(B_{-/+}=35 \approx 4380 / 127\right)$ when all examples from both classes are used, the dash-dot line). The standard deviations are shown as vertical bars.

An inspection of plots brings a number of interesting observations:

1. The un-balanced 2-class machines (dash-dot lines) and negative 1-class machines (dot lines) have inferior performance relative to either positive 1-class machines or the balanced 2-class SVMs for most values of $C$ (excepting very low $C$ ). Thus only the last two modes will be used in further research in this paper. 

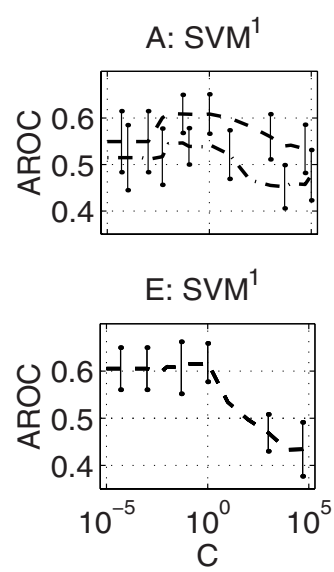

B: hSVM ${ }^{1}$

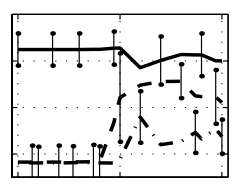

F: hSVM ${ }^{1}$

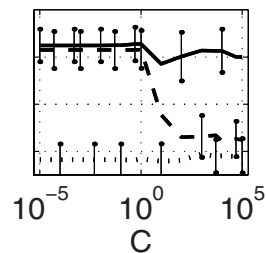

C: hSVM

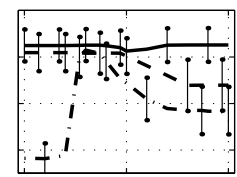

G: hSVM²

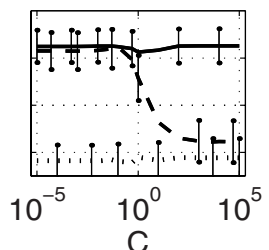

$\mathrm{D}: \mathrm{hRN^{2 }}$

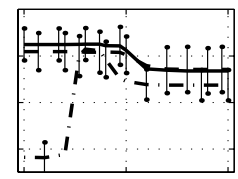

$\mathrm{H}: \mathrm{hRN}^{2}$

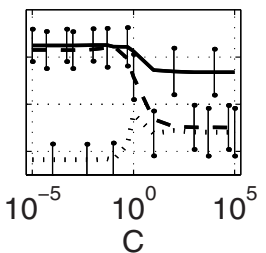

Fig. 1. Mean AROC for AHR-data as a function of the regularisation constant $C$ for 1-class and 2-class SVMs with the hard balancing (figures A-D) and the soft balancing (figures E-H) for four linear $(d=1)$ SVMs and four different modes: $(i)$ positive 1class $\left(B_{-/+}=0\right.$ and $B=+1$, solid line); ( $\left.i i\right)$ negative 1-class $(B=-1$, dotted line); (iii) balanced 2-class $\left(B_{-/+}=1\right.$ and $B=0$, dashed line); (iv) un-balanced 2-class $\left(B_{-/+}=35\right.$, the dash-dot line). The standard deviations are shown as vertical bars.

2. All positive 1-class and balanced 2-class machines show a very good and roughly equal performance for very low values of $C$. Additionally, these values of mean AROC are equal to that for the positive 1-class centroid $(B=+1$, $\mathrm{AROC}=62.4 \pm 3.4)$ and the balanced 2-class centroid $C n t r_{0}(B=0, \mathrm{AROC}=$ $61.5 \pm 3.9)$ trained on the whole data. In the case of soft balancing this can be inferred from Theorem 1 since it implies that the orders imposed on test data by the scores of the respective centroid and SVM classifiers coincide hence yield the same AROC (uniquely determined by such an order).

3. There are noticeable differences between the performance of different SVMs. For instance, note the differences between unbalanced 2-class $h S V M^{1}$ and $S V M^{1}$ (dash-dot lines in Figures 1]A and 1B, respectively).

4. Positive 1-class $h S V M^{2}$ is very robust across the whole range of $C$ values (cf. the solid line in Figure 1]C). In particular, for high values of $C$, i.e, virtually the hard margin case [8], it performs better than any other SVM tested. This setting was used for the winning submission to KDD Cup 2002.

In summary, the top performance by SVMs is achieved at extremely high values of $C$, i.e. hard margin case, or at the limit of very low $C$. For low $C$ the best SVMs are equivalent to respective centroid machines, for positive one-class $(B=1)$ or the balanced 2 class $(B=0)$. For the high $C$ s, the positive oneclass consistently outperforms other settings. This motivates our restrictions on experimental settings for the rest of the paper as follows. We shall concentrate exclusively on hard balanced SVMs trained with high $C$ s and centroid classifiers with $B$ set to $0\left(C n t r_{0}\right)$ for a range of hard balance mixture ratios $B_{-/+}$. 


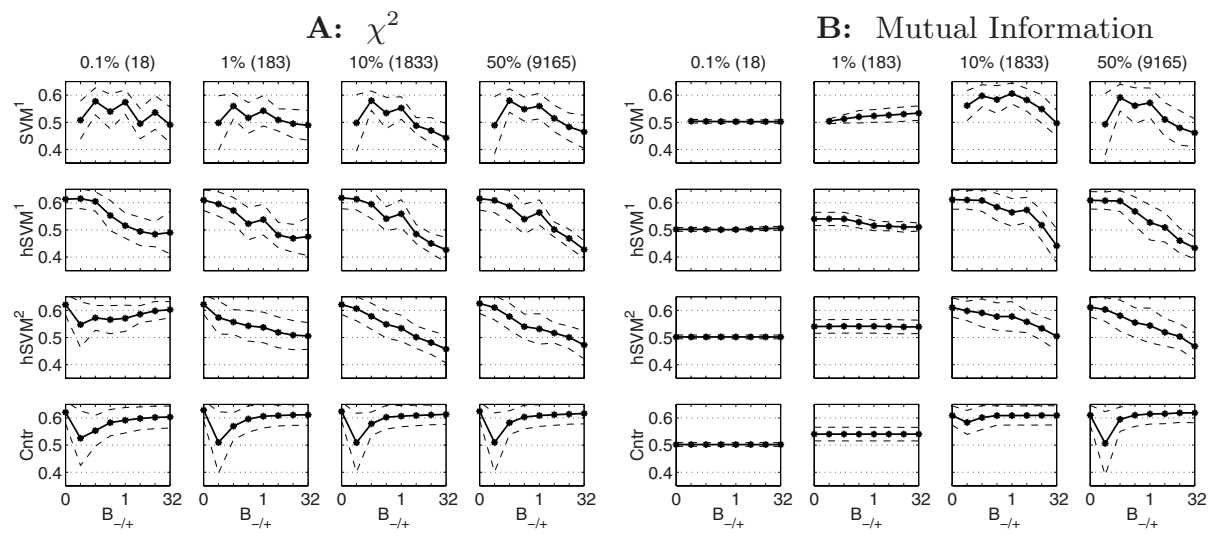

Fig. 2. Mean AROC with \pm standard deviation envelopes for AHR-data as a function of the hard balanced data with mixture ratio $B_{-/+} \in\{0,0.01,0.1,0.5,1,5,10,35\}$ for four different fractions of the original feature set $(0.1 \%, 1 \%, 10 \%$ and $50 \%$ out of 18,330 features). The three linear $(d=1)$ kernel machines were trained with $C=1000$, while the centroid classifiers were developed with $B=0$.

Impact of Feature Selection. We have used several different feature selection strategies including the document frequency thresholding [13], $\chi^{2}$ [18], mutual information [18, information gain [12], inverse document frequency - term frequency [13] and average discrimination scoring [13].

The results obtained have very similar trends, and due to space limitations we present in Figure 2 plots for only two selection methods: $\chi^{2}$ and mutual information. The main thing to observe is the trend of the dropping performance by SVMs as the negative class examples are added. For $h S V M^{1}$ this is visible even with 18 features selected by $\chi^{2}$ method. (This is also the case for all the other methods we have tested except mutual information.) The poor performance of mutual information at low fractions of features is exceptional among the techniques we have tested. It can be explained by a strong influence of this criterion by the marginal probability of features which tends to favour "rare" features rather than common ones.

The performance of $S V M^{1}$ is generally poorer than that of $h S V M^{1}$ or $h S V M^{2}$ at the same settings. Further, there are peaks and valleys at different mixture ratios that warrant further investigation into the behaviour of $S V M^{1}$.

The performance of the centroid classifiers $C n t r_{0}$ is quite different. There is a pronounced dip with high variance when very few negative examples are used $\left(B_{-/+} \leq 0.05\right.$, which represents 1-6 negative examples only). However, as more negative examples are included, the performance improves and catches up with that of the positive 1-class classifier. Further, this pattern is consistent even at extremely low number of features. 


\subsection{Experiments with Synthetic Data}

We observed in Section 4.1 that even in low dimensional space, the phenomenon of better performance with one-class learner persists. Our intuitive explanation here is that if the learner uses the minority class examples only, the "corner" (the half space) where minority data resides is properly determined. However, the minority class is "swamped" by the background class, hence once the background instances are added, the SVM solution becomes suboptimal. Now we explore this intuition using synthetic data.

We use three data sets of instances of similar structure. The observation vectors in these synthetic data sets contain a small number $n_{\text {inf }}$ of informative attributes and the remaining, larger number, $n_{\text {noise, }}$ of noise attributes. These attributes are binary, generated according to uniform random distribution with probabilities $P_{\text {inf }}$ and $P_{\text {noise }}$ of value $=+1$, respectively. The informative attributes determine the labels modulo the additional label noise which is the random reversal of certain proportions of labels, namely the proportions $L N_{+}$of the positive and $L N_{-}$of the negative labels. In all sets, we generate $m=9000$ instances of which $p_{y=+1}=3 \%$ have labels $y=+1$.

$-S_{1}$ : For this data set we use $n=n_{\text {inf }}+n_{\text {noise }}=1+999$ dimensions and $P_{\text {noise }}=2 \%$. The labels are generated as a random bipolar label vector $y \in\{ \pm 1\}^{9000}$ with the proportion $p_{y=+1}=3 \%$ of positive examples. For the informative dimension we set $x_{\text {inf }}=(y+1) / 2 \in\{0,1\}$ and then change randomly the proportion $L N_{-}=20 \%$ of 0 s to $1 \mathrm{~s}$.

$-S_{2}$ : In this case $n_{\text {inf }}=10, n_{\text {noise }}=990, P_{\text {inf }}=5 \%, P_{\text {noise }}=2 \%$. Having defined informative attributes $x_{i n f, i} \in \mathbb{R}^{10}$ for $i=1, \ldots, 9000$, we have randomly generated a vector $v \in \mathbb{R}^{10}$, then chosen a bias $b \in \mathbb{R}$ such that for $2004(\approx 22 \%)$ instances $i$ we got the scores $x_{i n f, i} \cdot v>b$. Of these 2004 instances, we randomly select 270 instances $(=3 \%$ of 9000) and label them +1 and the remaining 8730 instances we labelled -1 .

$-S_{3}$ : This set was designed to test the impact of non-linear kernels. It is generated as $S_{1}$ with the difference that only $n=n_{\text {inf }}+n_{\text {noise }}=1+19=20$ dimensions are used and the random proportions $L N_{+}$and $L N_{-}$of the both +1 and of 0 entries, respectively, are reversed in the second phase of the generation of the informative attribute $x_{\text {inf }}$.

In experiments, each set of 9000 instances generated as described above, was split randomly into 3000 training and 6000 test instances, with proportional sampling (without replacement) from both classes. All results reported are averages of 20 such random splits.

Figure 3 presents the results of experiments evaluating AROC as a function of the mixture ratio $B_{-/+}$, for the four kernel machines. For all three data sets, we show the results for the linear kernel (Figures $3 \mathrm{~A}-3 \mathrm{C}$ ), and for $S_{3}$ we show the impact of higher degree polynomial kernels (Figures $3 \mathrm{D}-3 \mathrm{~F}$ ).

The results, especially for $h S V M^{1}$, strikingly resemble those obtained for the AHR data (c.f Figure 2), with the consistent pattern of decreasing performance with increasing proportion of negative class instances. Note the 'collapse' of 

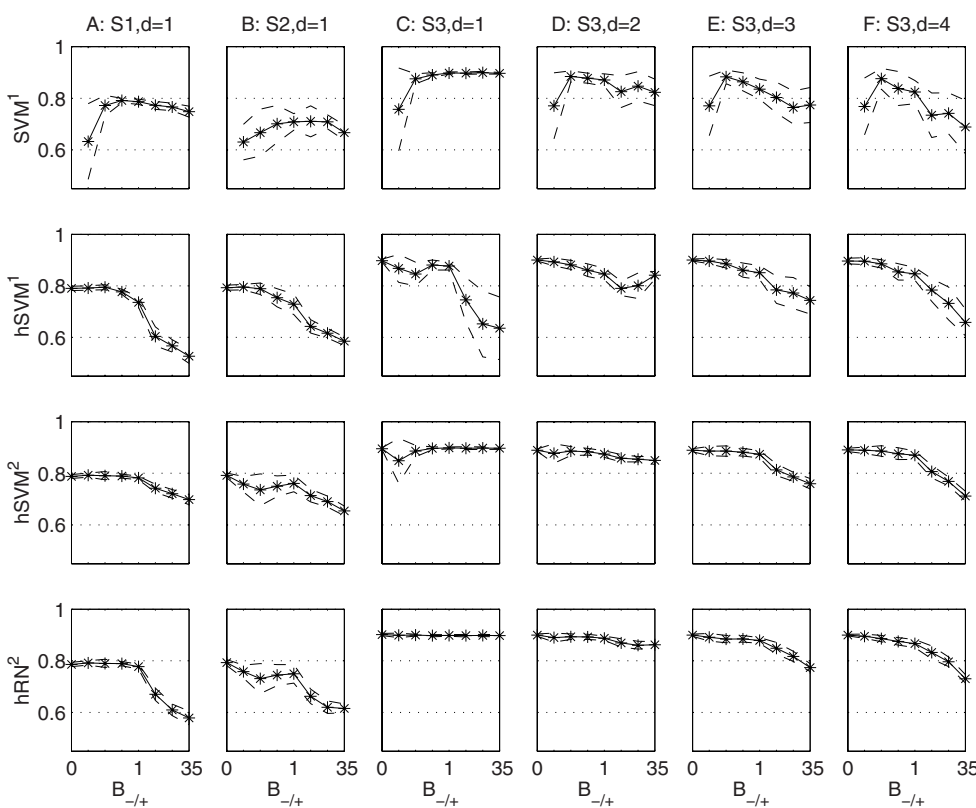

Fig. 3. Mean AROC with \pm standard deviation envelopes as a function of the mixture ratio $B_{-/+}$for four machines with $C=1000$ : $S V M^{1}, h S V M^{1}, h S V M^{2}$ and $h R N^{2}$. Plots A, B, C show results for linear kernels with $S_{1}, S_{2}$ and $S_{3}$, respectively. Results with higher degree polynomial kernels $(d=2,3,4)$ are shown for $S_{3}$ in plots D, E and $\mathrm{F}$, respectively. $B_{-/+}=[0,0.01,0.1,0.5,1,5,10,35]$.

$S V M^{1}$ algorithm when negative class proportion is very low and significantly better performance of $h S V M^{1}$ than $S V M^{1}$ for data dominated by the positive class $\left(B_{-/+} \approx 0\right)$ and reverse of it for data dominated by the negative class $\left(B_{-/+} \approx 1\right)$.

As kernel degree increases we observe the familiar pattern of decreasing performance with increasing dominance of negative class instances (Figures $3 \mathrm{C}\{3 \mathrm{~F}$ ). Thus, the relatively low dimensional $S_{3}$ data set when used with higher degree polynomial kernels behaves in a way similar to that of the high dimensional datasets $S_{1}$ and $S_{2}$ with linear kernels.

We also experimented with different values of the regularisation constant $C$, but found that this had marginal impact on AROC in the above settings.

In addition, our experiments with different label noise settings $\left(L N_{+}\right.$and $L N_{-}$) show that the pattern of decreasing performance with increasing amounts of negative class instances persists with different levels of label noise.

\section{Discussion}

It is interesting that in our extensive experiments, while positive one-class classifiers using $\approx 3 \%$ of the AHR-data provide best models, learning with negative class examples only provides very poor models (eg., mean AROC $=62.4 \pm 3.4 \%$ 
vs. $38.3 \pm 3.3 \%$ for centroid models and $h S V M^{1}, h S V M^{2}$ and $h R N^{2}$ classifiers at the low $C$ limit). Further, the unbalanced 2-class SVMs perform consistently below the level of the trivial random classifier $A R O C<50 \%$. The reasons for this behaviour need to be investigated more thoroughly.

Our experiments indicate that performance of one class learning method is dependent on learning machine, though the dominant tendency of performance deterioration with increased influence of the majority (the negative) class was visible for our range of classifiers. In particular, the popular support vector machine with linear penalty $\left(S V M^{1}\right)$ performed poorly for minority class dominated learning while performing much better for the majority class learning setting. The minor modification of this algorithm $\left(h S V M^{1}\right)$ has demonstrated quite opposite properties, while the support vector machine with quadratic penalty $\left(h S V M^{2}\right)$ performed relatively well over the whole range of settings. This appraisal of the positive one-class $h S V M^{2}$ holds true in particular, for its performance on AHR-data, where learning is implemented with 85 minority class instances in the full 18330 dimensional feature space.

The degradation of learning performance in the presence of abundant negative examples has been noted in [9]. Their solution of focusing on the best positive region works in low dimensional input space when there is a single region to be labelled as positive and the minority class is around $10 \%$ of the data. For our situation of very high dimensional input space with around $3 \%$ minority class data, the more drastic solution of totally ignoring negative class examples seems to work better for all machines.

Further, even when the sparse high dimensional space is reduced to a more dense representation via aggressive feature reduction methods, the advantage of one-class learners persist. This indicates that there is a combination of factors involved in this phenomenon, and a more thorough investigation with synthetic data is warranted.

Experiments with the polynomial kernels seem to indicate that interactions between the 19 noisy attributes in the set $S_{3}$ are equivalent to explicit addition of hundreds of extra noise attributes in the datasets $S_{1}$ and $S_{2}$. The higher the degree of the kernel, the more such 'noisy' virtual attributes are added (on the level of the feature space) and the more pronounced is the difference between one-class and two-class learning. Note that in this case, in contrast to the case of AHR-data case, the range of AROC values is around 60-90\%.

\section{Conclusion}

We have shown that learning from positive examples only can be advantageous for real life data such as AHR-data used in KDD Cup 2002 in term of classifier accuracy but is not restricted to this data set. A few synthetic data sets tested in this paper show that favourable conditions for such learning method can naturally arise in many other situations, in particular when popular support vector machines with non-linear kernels are used. More research is required to study these conditions. 
Our experiments demonstrate that one-class learning from positive class examples can be a very robust classification technique when dealing with very unbalanced data and high dimensional noisy feature space. It can be used as an alternative to aggressive feature selection usually used in such situations and can be very attractive for learning with non-linear kernels, when direct feature selection on the feature space level cannot be implemented.

\section{Acknowledgements}

The permission of the Managing Director, Telstra Research Laboratories, to publish this paper is gratefully acknowledged.

\section{References}

1. D. Bamber. The area above the ordinal dominance graph and the area below the receiver operating characteristic graph. J. Math. Psych., 12:387 - 415, 1975.

2. R. Centor. The use of ROC curves and their analysis. Med. Decis. Making, 11:102 106, 1991.

3. Y. Chen, X. Zhou, and T. Huang. One-class svm for learning in image retrieval. In Proceedings of IEEE International Conference on Image Processing (ICIP'01 Oral), 2001.

4. M. Craven. The Genomics of a Signaling Pathway: A KDD Cup Challenge Task. SIGKDD Explorations, 4(2), 2002.

5. N. Cristianini and J. Shawe-Taylor. An Introduction to Support Vector Machines and other kernel-based learning methods. Cambridge University Press, Cambridge, 2000 .

6. F. Girosi, M. Jones, and T. Poggio. Regularization theory and neural networks architectures. Neural Computation, 7(2):219-269, 1995.

7. N. Japkowicz and S. Stephen. The class imbalance problem: A systematic study. Intelligent Data Analysis Journal, 6(5), 2002.

8. A. Kowalczyk and B. Raskutti. One Class SVM for Yeast Regulation Prediction. SIGKDD Explorations, 4(2), 2002.

9. M. Kubat, H. R., and S. Matwin. Learning when negative examples abound. In Proceedings of the Ninth European Conference on Machine Learning ECML97, 1997.

10. D. Lewis and J. Catlett. Training Text Classifiers by Uncertainty Sampling. In Proceedings of the Seventeenth International ACM SIGIR Conference on Research and Development in Information Retrieval, 1994.

11. L. M. Maneivitz and M. Yousef. One-class SVMs for Document Classification. Journal of Machine Learning Research, 2:139-154, 2002.

12. J. R. Quinlan. Induction of Decision Trees. Machine Learning, 1(1), (1986).

13. G. Salton and M. J. McGill. Introduction to Modern Information Retrieval. McGraw Hill, 1983.

14. B. Schölkopf, J. Platt, J. Shawe-Taylor, A. Smola, and R. Williamson. Estimating the support of a high-dimensional distribution, 1999.

15. B. Schölkopf and A. J. Smola. Learning with Kernels: Support Vector Machines, Regularization, Optimization and Beyond. MIT Press, 2001. 
16. V. Vapnik. Statistical Learning Theory. Wiley, New York, 1998.

17. G. Whaba. Support vector machines, reproducing Hilbert spaces and the randomised GACV. In B. Schölkopf, C. Burges, and A. J. Smola, editors, Advances in Kernel Methods, pages 69-88, Cambridge, Ma., 1999. MIT Press.

18. Y. Yang and J. O. Pedersen. A Comparative Study on Feature Selection in Text Categorization. In Proceedings of the Fourteenth International Conference on Machine Learning, 1997. 